\title{
Multi-Color Imaging of Magnetic Co/Pt Multilayers
}

\author{
D. Weder ${ }^{1}$, C. Von Korff Schmising ${ }^{1}$, F. Willems ${ }^{1}$, C. M. Günther ${ }^{2}$, M. Schneider ${ }^{1}$, B. Pfau ${ }^{1}$, A. Merhe ${ }^{3,4}$, \\ E. Jal ${ }^{3,4}$, B. Vodungbo ${ }^{3,4}$, J. Lüning ${ }^{3,4}$, B. Mahieu ${ }^{5}$, F. Capotondi ${ }^{6}$, E. Pedersoli ${ }^{6}$, and S. Eisebitt ${ }^{1,2}$ \\ ${ }^{1}$ Max-Born-Institute Berlin, 12489 Berlin, Germany \\ ${ }^{2}$ Institut für Optik und Atomare Physik, Technische Universität Berlin, 10623 Berlin, Germany \\ ${ }^{3}$ Sorbonne Universités, UPMC Université Paris 06, UMR 7614, LCPMR, 75005 Paris, France \\ ${ }^{4}$ CNRS, UMR 7614, LCPMR, 75005 Paris, France \\ ${ }^{5}$ Laboratoire d'Optique Appliquée, ENSTA ParisTech, CNRS, Ecole Polytechnique, \\ Université Paris-Saclay, 91762 Palaiseau Cedex, France \\ ${ }^{6}$ Elettra-Sincrotrone Trieste, 34149 Trieste, Italy
}

We demonstrate for the first time the realization of a spatial resolved two color, element-specific imaging experiment at the free-electron laser facility FERMI. Coherent imaging using Fourier transform holography was used to achieve direct real space access to the nanometer length scale of magnetic domains of Co/Pt heterostructures via the element-specific magnetic dichroism in the extreme ultraviolet spectral range. As a first step to implement this technique for studies of ultrafast phenomena we present the spatially resolved response of magnetic domains upon femtosecond laser excitation.

Index Terms - Co/Pt, demagnetization, free-electron lasers (FEL), holography, magnetic domains, magnetic multilayers, perpendicular magnetic anisotropy, X-ray magnetic circular dichroism (XMCD).

\section{INTRODUCTION}

$\mathbf{U}$ LTRATHIN perpendicularly magnetized heterostructures, such as the two-compound multilayer system $\mathrm{Co} / \mathrm{Pt}$, show fascinating physical properties due to strong spin-orbit coupling [1]. Co/Pt multilayers have a significant interface contribution to the perpendicular magnetic anisotropy which can be used for the control of domain wall motion arising from an interplay of Dzyaloshinskii-Moriya interaction [2], [3] at the $\mathrm{Co} / \mathrm{Pt}$ interfaces and spin currents [4]. Also based on these mechanisms room-temperature skyrmions can be created in thin $\mathrm{Co} / \mathrm{Pt}$ films in racetrack memories [5]. Optical induced ultrafast spin currents in ferromagnetic thinfilm structures pave the way for future ultrafast spintronic devices [6] and broadband terahertz emitters [7]. Moreover, $\mathrm{Co} / \mathrm{Pt}$ thin-film magnetism has a huge potential in magnetic storage devices. Not only because of the fulfilled requirements of long-term stability due to the strong magnetic anisotropy but also because of recently discovered helicity-dependent all-optical control of ferromagnetic multilayers [8], [9]. However, many underlying microscopic mechanisms on ultrafast time and nanometer length scale are not fully understood and currently under debate. In particular, the origin of all-optical helicity-dependent switching or the fundamental microscopic processes leading to an optically induced ultrafast demagnetization on a femtosecond time the scale of ferromagnetic materials [10] raise many intriguing questions. To gain more insight into the interaction between non-local spin currents and interacting multi-compound perpendicularly magnetized

Manuscript received March 10, 2017; revised April 13, 2017; accepted April 19, 2017. Date of publication May 18, 2017; date of current version October 24, 2017. Corresponding author: D. Weder (e-mail: weder@mbi-berlin.de).

Color versions of one or more of the figures in this paper are available online at http://ieeexplore.iee.org.

Digital Object Identifier 10.1109/TMAG.2017.2699560 heterostructures requires novel techniques that give a direct and simultaneous view of the element-specific magnetization on a femtosecond time and nanometer length scale.

Here, we present the first experimental demonstration of two-color imaging with Fourier transform holography (FTH) in the extreme ultraviolet (XUV) spectral range measured at the free-electron laser (FEL) facility FERMI in Trieste, Italy. The reconstructed magnetic domain pattern gives simultaneously element specific and real space access to the magnetization of a two-compound $\mathrm{Co} / \mathrm{Pt}$ multilayer system. Furthermore, a time-resolved single-color approach reveals spatially resolved frames of the optical induced ultrafast demagnetization dynamics of $\mathrm{Co} / \mathrm{Pt}$. The feasibility and the prospect of combining both, multi-color spectroscopy with nanometer resolution and femtosecond spatial resolution are discussed.

\section{EXPERIMENTAL SETUP AND RESUlts}

The coherent imaging experiment was performed at the FEL facility FERMI at the end-station DiProI [11], [12] combining femtosecond temporal and nanometer spatial resolution in an optical pump-XUV probe setup. To image the magnetic domains of a $\mathrm{Co} / \mathrm{Pt}$ multilayer an FTH technique in transmission configuration [13]-[15] were used. In this imaging technique a hologram is formed by interference of an object with a known reference wave. In the XUV to soft X-ray spectral range this readily yields spatial resolutions on the order of approximately 30 to $80 \mathrm{~nm}$. For optimal stability the sample and the reference holes are manufactured in a monolithic mask design. The $250 \mathrm{~nm}$ thick gold mask with no transmission in the XUV spectral range is evaporated on a $30 \mathrm{~nm}$ thin silicon nitride membrane supported by a $200 \mu \mathrm{m}$ thick silicon substrate. A circular hole with a diameter of $d_{r}=$ $2 \mu \mathrm{m}$ is drilled in the mask via a focused ion beam and defines 


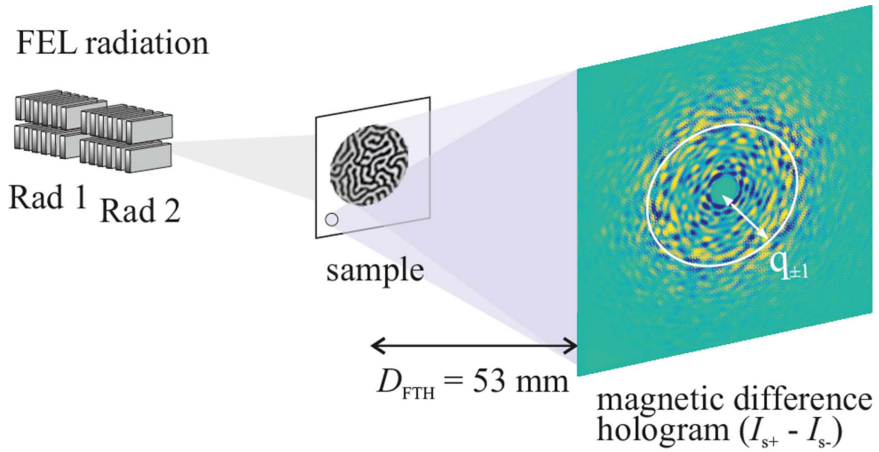

Fig. 1. Sketch of the FTH setup carried out at the FEL at FERMI. FEL radiation in the XUV spectral range is created in the undulators Rad 1 and 2, illuminating the $\mathrm{Co} / \mathrm{Pt}$ multilayer that is structured with a holographic gold mask. The diffracted radiation centered at the Co M-edge at $60 \mathrm{eV}$ originating from the reference wave and object wave interferes on a CCD detector placed $53 \mathrm{~mm}$ behind the sample. A beam stop cuts out the intense direct beam. The difference hologram between left and right circular polarization contains only magnetic information.

the object hole (field of view). Afterwards, the magnetic film $\mathrm{Al}(10) / \mathrm{Pt}(2) /[\mathrm{Co}(0.6) / \mathrm{Pt}(0.8)]_{20} / \mathrm{Al}(3) \mathrm{nm}$ is deposited on the opposite side of the membrane via magnetron sputtering. Due to prior sample characterizations via magnetic force microscopy and small angle X-ray scattering the domain size is determined to be on the order of $80 \mathrm{~nm}$. In the last step five reference holes with diameters ranging from 60 to $80 \mathrm{~nm}$ are drilled through the entire sample. Each reference will generate a twin-image of the object in the reconstruction leading to ten reference-object cross correlations. To increase the signal to noise ratio of the reconstruction, images stemming from the different reference holes can be added. To avoid an overlap of the reconstructed objects with the object-object or referencereference auto correlation in the center of the reconstruction, all references were arranged in a circle of $|\vec{r}|=5 \mu$ m diameter around the object hole, equidistantly placed with respect to each other.

The time-resolved single-color and static two-color coherent imaging experiment uses probe pulses in the XUV spectral range. The radiation is tuned to the Co M-edge and Pt N-edge for resonant small angle scattering due to the magnetic circular dichroism (MCD) effect. Magnetic sensitivity to Pt is confined to the $\mathrm{Co} / \mathrm{Pt}$ interface where band hybridization induces magnetism in Pt. The scattered intensity of reference and object holes form the interference pattern (hologram), which is recorded on a charge-coupled device (CCD) detector placed $53 \mathrm{~mm}$ behind the sample (Fig. 1). The difference image between holograms recorded with left/right circularly polarized radiation yields the magnetic information. To increase the dynamic range of the recordable intensity and to avoid damage to the CCD detector a beam stop was used to block the intense direct beam.

The achievable spatial resolution is determined by the size of the reference hole and the maximum-recorded scattering vector $q_{\max }$ which is determined by

$$
q_{\max }=\frac{4 \pi}{\lambda} \sin \theta_{\max }
$$

with the wavelength $\lambda$ and the maximum scattering angle $\theta_{\max }$ that is given by the size of the CCD chip and the
sample-CCD distance. The experimental setup enables a maximum recordable wave vector of $q_{\max }=77 \mu \mathrm{m}^{-1}$ at $20.8 \mathrm{~nm}$ (Co M-edge) and $q_{\max }=92 \mu \mathrm{m}^{-1}$ at $17.3 \mathrm{~nm}$ (Pt N-edge) leading to resolvable spatial frequencies of $d=2 \pi / q_{\max }=$ 82 and $68 \mathrm{~nm}$, respectively. Also note that in the XUV spectral range wave guiding effects of the reference hole may further influence the spatial resolution.

As a significant number of photons with large scattering angles are required, we average several hundreds to thousands of FEL pulses to form the hologram. At the same time, the XUV intensity of the FEL pulses in the imaging experiments has to be reduced to prevent non-reversible changes and damage of the magnetic domain network [16], [17].

\section{A. Time-Resolved Single-Color Imaging of Co/Pt}

For the time-resolved single-color imaging of $\mathrm{Co} / \mathrm{Pt}$ we optically excite with laser radiation centered at $800 \mathrm{~nm}$. The laser-triggered ultrafast dynamics are probed by coherent XUV radiation at the $\mathrm{M}$-edge of $\mathrm{Co}$ at $60 \mathrm{eV}$. The laser fluenc was set to approximately $3 \mathrm{~mJ} / \mathrm{cm}^{2}$. The pulselength of the optical pump beam and the XUV probe beam is on the order of $100 \mathrm{fs}$. For each helicity we integrated $10 \mathrm{~min}$ with a $10 \mathrm{~Hz}$ repetition rate, i.e., 6000 pulses.

In Fig. 2(a), we show the spatially resolved reconstruction of the magnetic domains in an unpumped state 1 ps before time zero. The clearly resolved magnetic domains with a known periodicity of $160 \mathrm{~nm}$ allow a conservative estimation of the spatial resolution below $80 \mathrm{~nm}$. Black and white areas represent out-of-plane worm-like domains pointing in and out of the surface while the surrounding gray area represents the border of the field of view. The cross section of the domain contrast (line) is displayed in more detail in Fig. 3(b). In Fig. 2(b), one can see the reconstructed domains within the very same field of view but in an excited state 1 ps after time zero. As the contrast settings of both images are scaled in the same way a direct comparison between the pumped and unpumped domain pattern is possible. The excited $\mathrm{Co} / \mathrm{Pt}$ film shows a strongly reduced magnetic domain contrast caused by ultrafast demagnetization of the up and down domains. This is further emphasized by the direct comparison of the cross sections of the unpumped and pumped contrast, shown in solid and dashed-dotted lines, respectively, in Fig. 3(b). To investigate whether the magnetic domain pattern shows any non-reversible changes either due to the FEL probe or the optical excitation pulses, we subtract the scaled pumped reconstruction from the unpumped reconstruction. The scaling factor is determined with a spatially resolved minimization algorithm and yields 2.4, corresponding to a global demagnetization rate of $\sim 42 \%$. The result is depicted in Fig. 3(a) and shows an almost perfect difference image and no indication of large magnetic domain reconfiguration or nonuniform demagnetization. Due to the high spatial resolution and the high sensitivity for the magnetization, it is possible to identify and localize small changes of the domain network and, if required, take them into consideration in the data evaluation. Careful inspection of Fig. 3(a) suggests that we can indeed identify small areas of non-reversible changes, see black and white dots in Fig. 3(a). This analysis is expected 

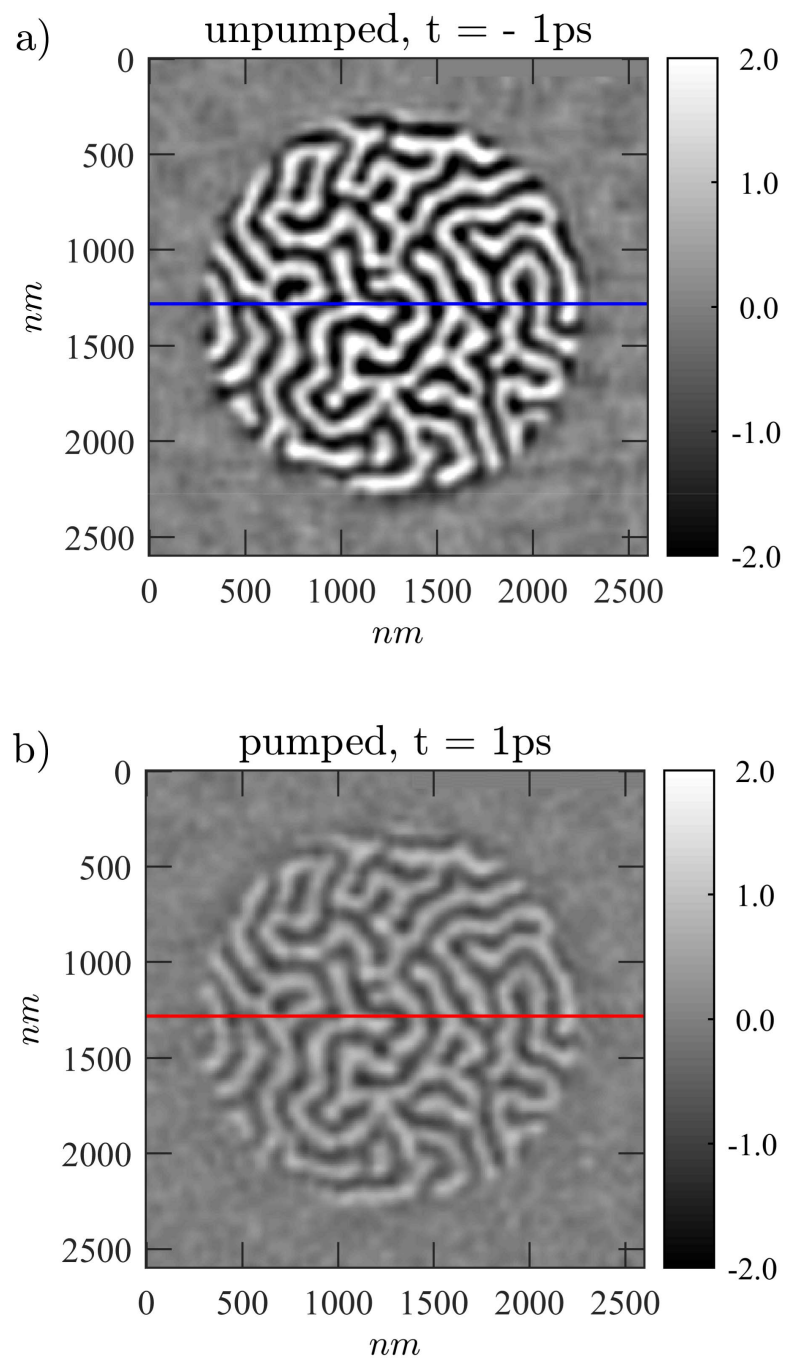

Fig. 2. (a) Difference holograms of an unpumped and (b) pumped magnetic domain pattern can be reconstructed with the help of a Fourier transformation. The result is visible in the two top panels showing a worm-like domain network within a field of view of $2 \mu \mathrm{m}$ diameter.

to be particularly powerful in nano-structured samples where multiple reflections and interference of the incident pump light with the subwavelength dimension of the sample leads to an inhomogeneous intensity distribution. Controlling such complex light-matter interaction in nanostructures may allow to induce tailored absorption for nanoscale confinement of optically induced magnetization dynamics [12], [18].

Time resolved single-color imaging of $\mathrm{Co} / \mathrm{Pt}$ using the MCD contrast mechanism is a sensitive and powerful tool for imaging magnetic heterostructures on a nanometer length and ultrafast time scale without inducing permanent changes or damaging their magnetic properties. That makes it the ideal tool for repetitive investigations of domain wall motion, skyrmion dynamics, and helicity-dependent all-optical switching in real space.

\section{B. Static Multi-Color Imaging of $\mathrm{Co} / \mathrm{Pt}$}

For simultaneous probing of the element-specific response of the magnetization of the Co film and the Co/Pt interface, we performed a static two-color imaging. This is possible due
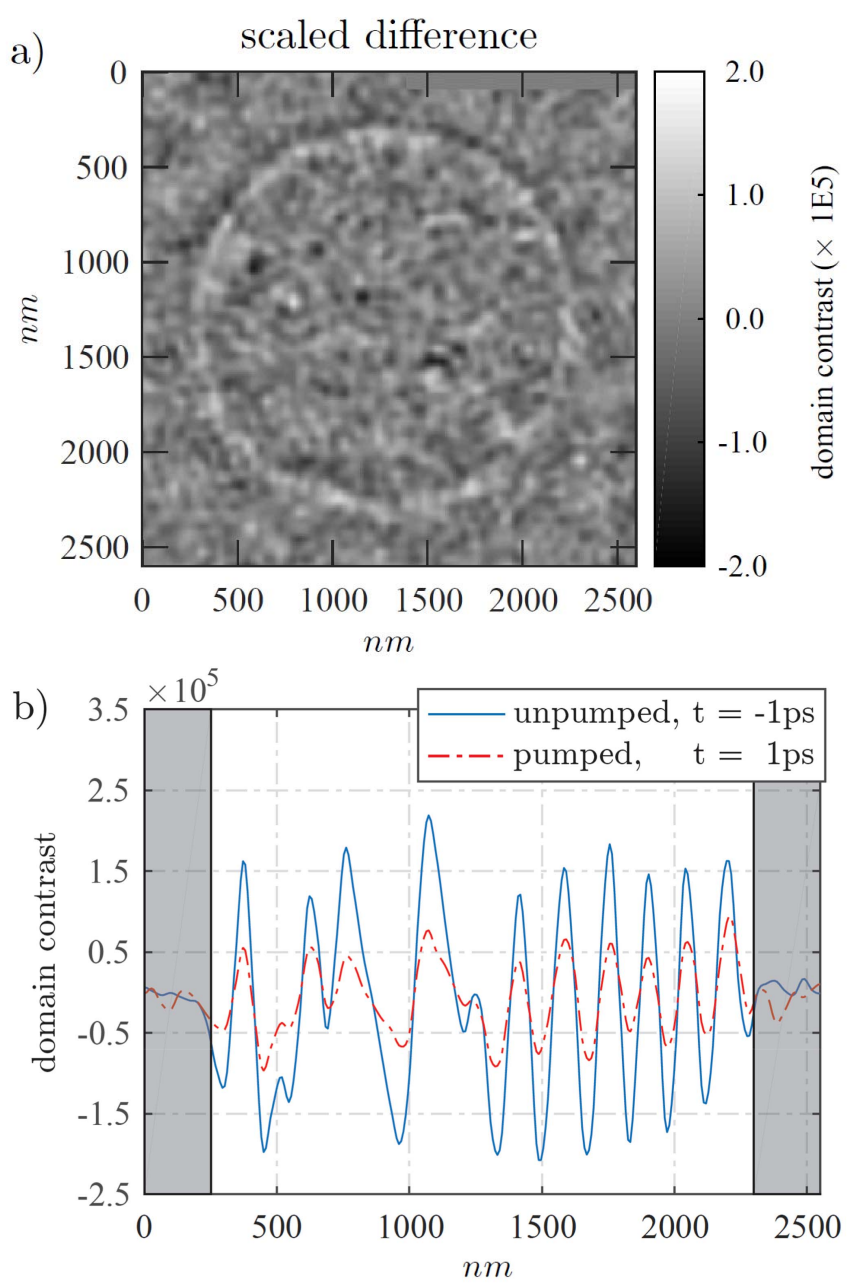

Fig. 3. Scaled difference of both reconstructions in (a) yields spatially resolved information about optical induced permanent changes of the domain structure. To compare the magnetic domain contrast of the $\mathrm{Co} / \mathrm{Pt}$ multilayer between the excited (dashed-dotted lines) and not excited (solid line) state (b), two lineouts are plotted over the spatial coordinates. They not only show a stable domain position but also indicate that the amplitude of magnetization is quenched by approximately $40 \%$.

to the recent achievement at FERMI to produce two-color FEL radiation at integer multiples of the UV seed laser [19].

An experiment at the synchrotron facility BESSY II revealed pronounced dichroic signals in the XUV spectral range stemming from $\mathrm{Co}$ and the $\mathrm{Co} / \mathrm{Pt}$ interface [20]. MCD of comparable amplitude was found at $61.4 \mathrm{eV}$ (20.2 nm) slightly off resonant at the Co $\mathrm{M}_{2,3}$ edge and at $71.6 \mathrm{eV}(17.3 \mathrm{~nm})$ at the $\mathrm{Pt} \mathrm{N}_{7}$ edge. These FEL energies can be achieved by seeding the FEL with UV light at $242.2 \mathrm{~nm}$ and tuning the first part of the undulator, $\operatorname{Rad} 1$, to the 12 th harmonic to probe $\mathrm{Co}$ and the second part of the undulator, $\operatorname{Rad} 2$, to the 14th harmonic to probe Pt.

For the physical dimensions of the holography mask containing an object hole with a diameter of $d_{r}=2 \mu \mathrm{m}$ and an object-reference distance of $|\vec{r}|=5 \mu \mathrm{m}$, one expects an overlap of the two reconstructions for the two respective wavelengths of 17.3 and $20.2 \mathrm{~nm}$. To ensure that both reconstructions pertaining to $\mathrm{Co}$ and $\mathrm{Pt}$ do not spatially overlap, one can either reduce the size of the field of view along the dispersion direction $\vec{r}$ or increase the distance between object 


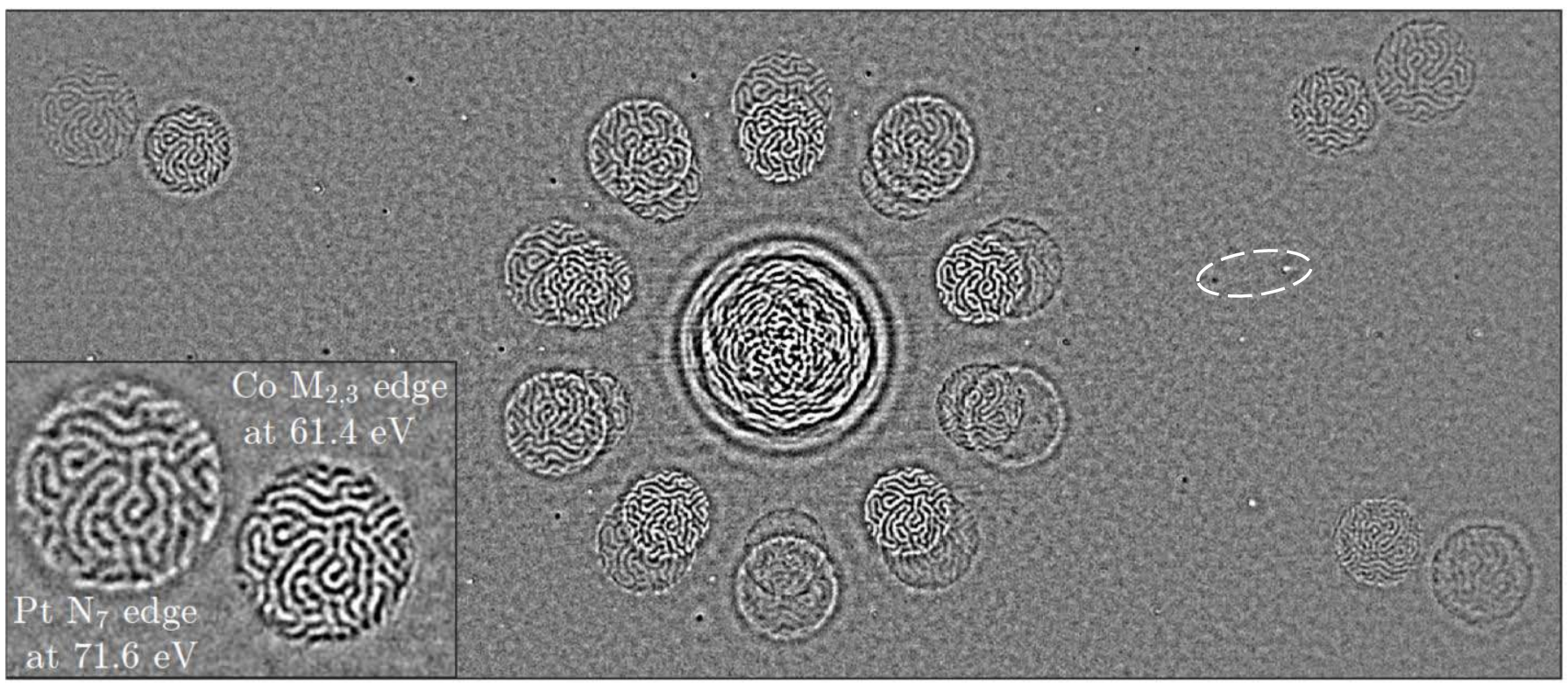

Fig. 4. Reconstruction of the static multi-color hologram of Co/Pt. All auto correlations are overlapped in the middle of the image. They are surrounded by a ring of 20 reconstructions of the object originating from the cross correlations between object and the five references for the two distinct energies. The two additionally references with $|\vec{r}|=13 \mu \mathrm{m}$ lead to four clearly separated reconstructions of the magnetic domains coming from Co and the Co/Pt interface. For a more detailed depiction see the magnified inset. The small dots that are distributed over the reconstruction (see dashed white line for example) stemming from the cross correlations between the references sized between 60 and $80 \mathrm{~nm}$.

and reference holes. More concretely, we have to fulfill the condition $|\vec{r}|>d_{r} \lambda / \Delta \lambda$. Since the FEL beam has a sufficient transverse coherence length, we add two additional reference holes at $|\vec{r}|=13 \mu \mathrm{m}$.

The energy of the two FEL pulses was set to $2 \pm 0.3 \mu \mathrm{J}$ at $61.4 \mathrm{eV}$ and $3 \pm 0.3 \mu \mathrm{J}$ at $71.6 \mathrm{eV}$. With a spot size of 180 $\mu \mathrm{m} \times 190 \mu \mathrm{m}$ (full width at half maximum) this corresponds to $8 \times 10^{6}$ photons $/ \mu \mathrm{m}^{2} /$ pulse and $10^{7}$ photons $/ \mu \mathrm{m}^{2} /$ pulse at the $\mathrm{Co}$ and $\mathrm{Pt}$ resonance, respectively. By adding up five images in $10 \mathrm{~Hz}$ mode with an integration time of $600 \mathrm{~s}$ for left and right helicity we obtain a hologram containing charge and magnetic contribution. A Fourier transformation of the difference hologram yields the direct visualization of a real space, element-specific and simultaneously recorded image of the magnetic domain network of Co and Pt with a high spatial resolution on the order of $80 \mathrm{~nm}$.

The very first reconstruction of such a simultaneously probed two-compound system is shown in Fig. 4. It shows the Fourier transformation of a single difference hologram and reconstructs the magnetic domains from Co and from the $\mathrm{Co} / \mathrm{Pt}$ interface. In the center of the reconstruction, all auto correlations (object-object and reference-reference) are located. They are surrounded by the cross correlations between object and reference holes. Note that we can also readily identify the cross correlations of the different reference holes showing up as white/black spots in the reconstruction. The fact that we can easily resolve them corroborates our conservative estimate of our spatial resolution on the order of $80 \mathrm{~nm}$. As the position of the reconstructed object is energy dependent [21] we observe two displaced fields of view stemming from the two distinct XUV wavelengths. The well-separated cross correlations at the four edges originate from the two additional reference holes and the object. In the lower left corner, we show a magnified depiction of the element-specific reconstruction. Here we have increased the signal to noise ratio by summing up the two independent reconstructions from the two new reference holes. A careful comparison of the reconstructions from $\mathrm{Co}$ and from $\mathrm{Pt}$ yields an identical but inverted domain contrast. This is due to an opposite sign of the MCD effect of the Co M-edge $(61.4 \mathrm{eV})$ and Pt N-edge $(71.6 \mathrm{eV})$, i.e., the magnetization of the Co film and the Co/Pt interface point in the same direction [22].

Using this method in a dynamic manner can lead to a deeper understanding of the influence of localized $\mathrm{Co} / \mathrm{Pt}$ interfaces on ultrafast demagnetization dynamics.

\section{CONCLUSION}

We demonstrated that single-color coherent imaging of $\mathrm{Co} / \mathrm{Pt}$ heterostructures via FTH in the XUV spectral range is a powerful tool to gain detailed information about magnetization dynamics on the nanometer length and ultrafast time scale. Furthermore, we presented the very first implementation of a two-color imaging at the FEL facility FERMI by taking advantage of the MCD effect at the Co M-edge and the strong dichroic signal stemming from the $\mathrm{Pt} \mathrm{N}$-edge. It allows simultaneous and element-specific access to thin-film magnetism. By going from the M-edge to the L-edge of Co and Pt one could push the limit of spatial resolution below $20 \mathrm{~nm}$ which for example allows direct imaging of magnetic skyrmions.

We point out the feasibility of combining both approaches for setting up a time resolved, simultaneous and multi-element probing to unravel ultrafast processes in thin magnetic multicompound heterostructures on a nanometer scale. 


\section{REFERENCES}

[1] T. Ueno et al., "Enhanced orbital magnetic moments in magnetic heterostructures with interface perpendicular magnetic anisotropy," Sci. Rep., vol. 5, Oct. 2015, Art. no. 14858. [Online]. Available: http://www. nature.com/articles/srep14858

[2] I. Dzyaloshinsky, "A thermodynamic theory of 'weak' ferromagnetism of antiferromagnetics," J. Phys. Chem. Solids, vol. 4, no. 4 pp. 241-255, Jan. 1958. [Online]. Available: http://www.sciencedirect. com/science/article/pii/0022369758900763

[3] T. Moriya, "Anisotropic superexchange interaction and weak ferromagnetism," Phys. Rev., vol. 120, no. 1, pp. 91-98, 1960. [Online]. Available: http://journals.aps.org/pr/abstract/10.1103/PhysRev.120.91

[4] K. S. Ryu, L. Thomas, S.-H. Yang, and S. Parkin, "Chiral spin torque at magnetic domain walls," Nature Nanotechnol., vol. 8, no. 7, pp. 527-533, Jun. 2013. [Online]. Available: http://www.ncbi.nlm.nih. gov/pubmed/23770808

[5] S. Woo et al., "Observation of room-temperature magnetic skyrmions and their current-driven dynamics in ultrathin metallic ferromagnets," Nature Mater., vol. 15, no. 5, pp. 501-506, 2106. [Online]. Available: http://arxiv.org/abs/-1502.07376\%5Cn and http://www.nature. com/doifinder/10.1038/nmat 4593

[6] T. J. Huisman et al., "Femtosecond control of electric currents in metallic ferromagnetic heterostructures," Nature Nanotechnol., vol. 11, pp. 455-458, Feb. 2016. [Online]. Available: http://www.nature. com/nnano/journal/v11/n5/full/nnano.2015.331.html

[7] T. Kampfrath et al., "Terahertz spin current pulses controlled by magnetic heterostructures," Nature Nanotechnol., vol. 8, no. 4, pp. 256-260, Mar. 2013. [Online]. Available: http://www.nature. com/nnano/journal/v8/n4/full/nnano.2013.43.html

[8] T. D. Cornelissen, R. Córdoba, and B. Koopmans, "Microscopic model for all optical switching in ferromagnets," Appl. Phys. Lett., vol. 108, no. 14, p. 142405, Apr. 2016. [Online]. Available: http:// aip.scitation.org/doi/full/10.1063/1.4945660

[9] C.-H. Lambert et al., "All-optical control of ferromagnetic thin films and nanostructures," Science, vol. 345, no. 6202, pp. 1337-1340, Sep. 2104. [Online]. Available: http://science.sciencemag. org/content/345/6202/1337

[10] E. Beaurepaire, J.-C. Merle, A. Daunois, and J.-Y. Bigot, "Ultrafast spin dynamics in ferromagnetic nickel," Phys. Rev. Lett., vol. 76, no. 22, pp. 4250-4253, May 1996. [Online]. Available: http://journals. aps.org/prl/abstract/10.1103/PhysRevLett.76.4250

[11] F. Capotondi, E. Pedersoli, N. Mahne, R. H. Menk, G. Passos, and L. Raimondi, "Invited article: Coherent imaging using seeded free-electron laser pulses with variable polarization: First results and research opportunities," Rev. Sci. Instrum., vol. 84, no. 5, p. 051301, May 2013. [Online]. Available: http://aip.scitation.org/doi/abs/10.1063/ 1.4807157
[12] C. V. K. Schmising et al., "Imaging ultrafast demagnetization dynamics after a spatially localized optical excitation," Phys. Rev. Lett., vol. 112, no. 21, p. 217203, May 2014. [Online]. Available: http://journals.aps.org/prl/abstract/10.1103/PhysRevLett.112.217203

[13] B. Pfau and S. Eisebitt, "X-ray holography," in Synchrotron Light Sources and Free-Electron Lasers, E. J. Jaeschke, S. Khan, J. R. Schneider, and J. B. Hastings, Eds. Cham, Switzerland: Springer, 2016, pp. 1093-1133.

[14] S. Eisebitt et al., "Lensless imaging of magnetic nanostructures by X-ray spectro-holography," Nature, vol. 432 , no. 7019 , pp. 885-888, Dec. 2004. [Online]. Available: http://www.nature.com/nature/journal/ v432/n7019/abs/nature03139.html

[15] S. Schaffert et al., High-resolution magnetic-domain imaging by Fourier transform holography at $21 \mathrm{~nm}$ wavelength. New J. Phys., vol. 15 , p. 093042, Sep. 2013. [Online]. Available: http://iopscience.iop.org/ article/10.1088/1367-2630/15/9/093042

[16] C. Gutt et al., "Single-pulse resonant magnetic scattering using a soft X-ray free-electron laser," Phys. Rev. B, Condens. Matter, vol. 81, no. 10, p. 100401, Mar. 2010. [Online]. Available: http://journals. aps.org/prb/abstract/10.1103/PhysRevB.81.100401

[17] T. Wang et al., "Femtosecond single-shot imaging of nanoscale ferromagnetic order in $\mathrm{Co} / \mathrm{Pd}$ multilayers using resonant $\mathrm{X}$-ray holography," Phys. Rev. Lett., vol. 108, no. 26, p. 267403 Jun. 2012. [Online]. Available: http://journals.aps.org/prl/abstract/ 10.1103/PhysRevLett.108.267403

[18] E. Allaria et al., "Two-colour pump-probe experiments with a twinpulse-seed extreme ultraviolet free-electron laser," Nature Commun. vol. 4, Sep. 2013, Art. no. 2476. [Online]. Available: http://www. nature.com/articles/ncomms 3476

[19] L. Le Guyader et al., "Nanoscale sub-100 picosecond all-optical magnetization switching in GdFeCo microstructures," Nature Commun. vol. 6, Jan. 2015, Art. no. 5839. [Online]. Available: http://www. nature.com/articles/ncomms6839

[20] F. Willems et al., "Multi-color imaging of magnetic Co/Pt heterostructures," Struct. Dyn., vol. 4, Jan. 2017, Art. no. 014301. [Online]. Available: http://aca.scitation.org/doi/10.1063/1.4976004

[21] B. Pfau et al., "Femtosecond pulse X-ray imaging with a large field of view," New J. Phys., vol. 12, Sep. 2010, Art. no. 095006. [Online]. Available: http://iopscience.iop.org/article/10.1088/13672630/12/9/095006/meta

[22] F. Willems et al., "Probing ultrafast spin dynamics with high-harmonic magnetic circular dichroism spectroscopy," Phys. Rev. B, Condens. Matter, vol. 92, Dec. 2015, Art. no. 220405. [Online]. Available: https://journals.aps.org/prb/abstract/10.1103/PhysRevB.92.220405 